\title{
Germanica
}

$47 \mid 2010$

« Krack! Tschock ! Pflatsch ! Bummmm ! La BD de langue allemande (à suivre...) »

\section{Struwwelpeter et Anti-Struwwelpeter . La bande dessinée au service des idéologies}

Struwwelpeter und Anti-Struwwelpeter

Struwwelpeter and the Anti-Struwwelpeter

\section{Marie-Hélène Quéval}

\section{OpenEdition}

\section{Journals}

Édition électronique

URL : http://journals.openedition.org/germanica/1124

DOI : 10.4000/germanica.1124

ISSN : 2107-0784

\section{Éditeur}

Université de Lille

\section{Édition imprimée}

Date de publication : 31 décembre 2010

Pagination : 87-98

ISBN : 9782913857261

ISSN : 0984-2632

\section{Référence électronique}

Marie-Hélène Quéval, «Struwwelpeter et Anti-Struwwelpeter. La bande dessinée au service des idéologies », Germanica [En ligne], 47 | 2010, mis en ligne le 01 décembre 2012, consulté le 06 octobre 2020. URL : http://journals.openedition.org/germanica/1124; DOI : https://doi.org/10.4000/ germanica. 1124

Ce document a été généré automatiquement le 6 octobre 2020.

(c) Tous droits réservés 


\section{Struwwelpeter et Anti-Struwwelpeter . La bande dessinée au service des idéologies}

Struwwelpeter und Anti-Struwwelpeter

Struwwelpeter and the Anti-Struwwelpeter

Marie-Hélène Quéval

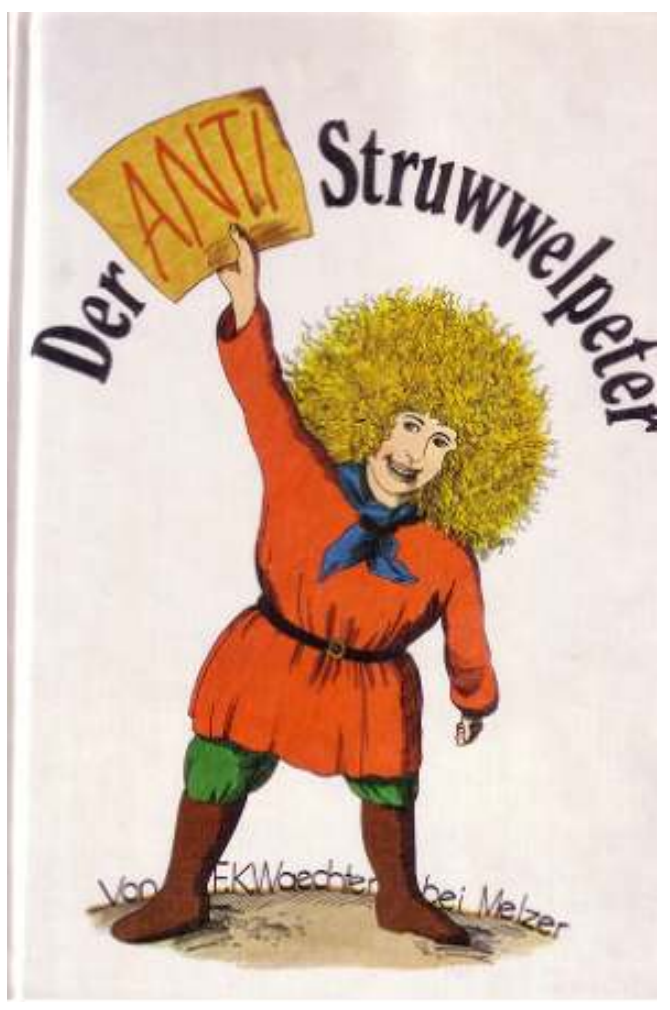

Dès la fin de la Seconde Guerre mondiale, une crise de la conscience déchire l'Allemagne et entraîne une transformation profonde des mentalités. Dans une 
première phase, la réflexion morale condamne en bloc la nation allemande grâce au concept chrétien de "culpabilité collective» (Kollektivschuld); les intellectuels s'interrogent : peut-on trouver une explication dans l'histoire, les traditions, ou encore les modes de vie, à la catastrophe du national-socialisme? Les sociologues, psychologues, pédagogues et philosophes donnent chacun un éclairage divers permettant de comprendre et de traiter le mal à la racine. À la fin des années 1960, la révolte des étudiants aboutit à un grand mouvement de libération souvent désigné par le chiffre fétiche de 1968 ; la jeunesse fustige les structures autoritaires de la société allemande et de sa plus petite cellule : la famille. Tous adhèrent au cri de rage lancé par André Gide dans les Nourritures terrestres : «Familles, je vous haie !»

2 C'est dans ce contexte qu'il faut situer la réécriture du Struwwelpeter ${ }^{1}$ que le médecin Heinrich Hoffmann (1809-1894) avait en 1844 dessiné et composé pour son fils malade alors âgé de 3 ans. Le livre connaît un succès incontesté jusque dans les années 1970 lorsque, pris dans la mouvance libertaire de la révolte étudiante, Friedrich K. Waechter (1937-2005), dessinateur et caricaturiste des revues gauchistes Pardon et Twen en réécrit une nouvelle version anti-autoritaire : Der Anti-Struwwelpeter ${ }^{2}$. Proche de la commune I (1967-1969), il partage son mépris des structures familiales et sociales accusées de brider l'imagination et la créativité naturelles à l'homme.

Une comparaison des deux œuvres fait apparaitre le renversement des valeurs qui a eu lieu dans la deuxième moitié du $\mathrm{xx}^{\mathrm{e}}$ siècle en Allemagne, le principe d'autorité étant remplacé par les droits de l'imagination créatrice.

\section{La contestation du principe d'autorité}

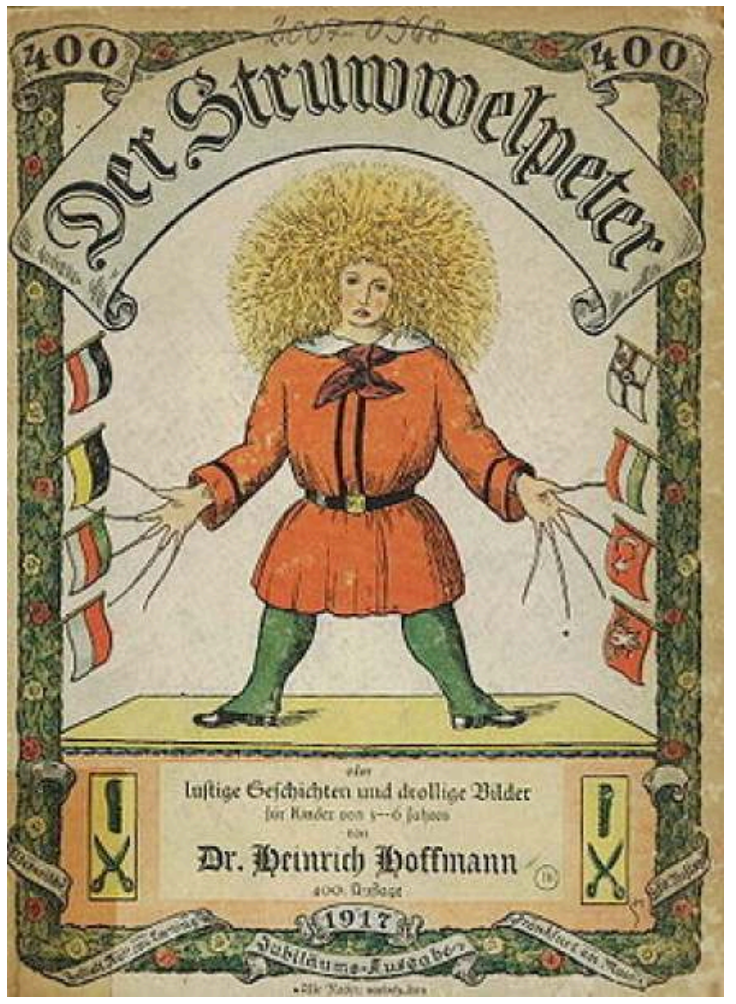

$4 \mathrm{Si}$, dans cette période pourtant stable sur le plan économique et politique, certains avec la RAF se lancent dans la lutte armée pour renverser un système ressenti comme 
aliénant, d'autres essaient plus modestement d'agir à leur niveau en remettant en cause les structures de l'entreprise ou de l'université, ou plus simplement celles de la famille considérée comme première source et premier lieu de l'aliénation; car, ils pensent, avec Wilhelm Reich (1897-1957) et Alice Miller (1923-), qu'elle perpétue l'autoritarisme et l'aveuglement idéologique des masses. Dans les années 1970, Michel Foucault (1926-1984) connaît à son tour un succès fulgurant avec son étude Surveiller et punir (1973) qui devient le livre de chevet des étudiants berlinois.

5 Reich, Miller et Foucault établissent une interrelation entre sexualité et politique. La répression par l'État autoritaire commence, disent-ils, par l'assujettissement du corps et la surveillance permanente de l'enfant, puis de l'adulte, par la famille et les éducateurs d'abord, par les institutions de l'État ensuite. Les exercices physiques, le sport et la gymnastique, les manœuvres et la formation militaires, les notations à l'école, l'attribution d'un rang et d'une place, les classements, les examens, tout contribue à soumettre l'enfant puis l'adolescent à une instance qui le juge, le classe, le condamne. L'adulte ne connaîtra le succès qu'à la condition d'intérioriser le code social.Dès le début de la vie, l'éducation met l'accent sur la soumission à une norme qu'on applique sans en comprendre le sens. Le but de l'État est de maîtriser, d'uniformiser la multiplicité humaine et de manipuler les forces vives de l'humanité. Terrible est le destin d'un des plus grands poètes allemands, Hölderlin. Comment ne pas frémir d'horreur en imaginant son visage enserré par le masque de son médecin tortionnaire Johann Hermann Heinrich Ferdinand von Autenrieth (1772-1835), ce masque qui symbolise l'enfermement et la répression du génie libre par définition. Pour Michel Foucault la fonction de la discipline est d'assujettir, de casser la volonté individuelle, de créer enfin le «nous» anonyme dans lequel se fond l'individu désormais privé de volonté propre.

6 Les lieux de la discipline sont d'abord l'hôpital psychiatrique et la prison, mais aussi l'école, l'usine et l'armée. Foucault les compare au Panoptique de Jeremy Bentham (1748-1832) qui dès 1791 servit de modèle aux prisons victoriennes, plus tard même au pénitencier de Cuba. Le principe était de placer au centre de la prison construite en cercle, une tour de contrôle dont les fenêtres, rendues aveugles par des persiennes, empêchaient le prisonnier de voir si quelqu'un se trouvait dans la salle de garde.Le gardien pouvait observer toutes les cellules sans lui-même être vu par les prisonniers soumis ainsi à une vigilance de tous les instants. Le panoptique symbolise pour Foucault le fonctionnement automatique du pouvoir. Plus tard, le penseur polonais Zygmunt Bauman (1925) déclarera que la postmodernité correspond à une époque postpanoptique, car à l'ère de l'Internet et du téléphone portable, la surveillance absolue et universelle ne dépend plus d'un lieu.Dans la deuxième moitié $\mathrm{du} \mathrm{xx}^{\mathrm{e}}$ siècle, la vision développée par Georges Orwell dans son roman 1984 semble s'être réalisée avec l'installation de caméras de surveillance dans les lieux publics tels que le métro, les gares, les magasins, les places publiques, les rues mêmes. Le pouvoir devient mécanique; c'est, comme le formule Michel Foucault, une

machine à créer et à soutenir un rapport de pouvoir indépendant de celui qui l'exerce ${ }^{3}$. 


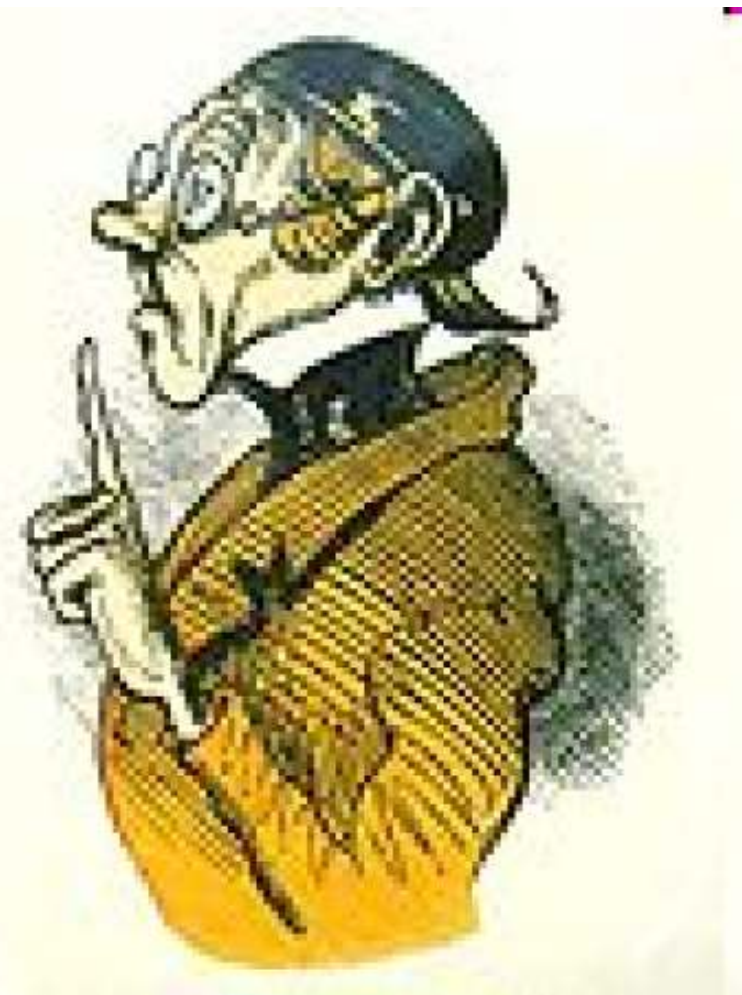

7 Désindividualisé, désincarné, automatisé, le pouvoir est devenu invisible comme celui que symbolise la tour de contrôle imaginée par Bentham : on est totalement vu, sans jamais voir.

8 Née en 1923 en Pologne, Alice Miller ${ }^{4}$ critique l'éducation par la peur. Miller a été frappée par les cas d'Adolf Eichmann (1906-1962) et de Rudolf Höss (1900-1947), deux petits garçons modèles. Le cas de Höss lui semble particulièrement étrange : enfant de chœur, bon catholique, bon élève, Höss se destinait à la carrière ecclésiastique. Comment expliquer que ce futur prêtre ait pu devenir l'implacable commandant d'Auschwitz? Miller l'explique par une pédagogie fondée sur la terreur et la soumission ; habitué à exécuter des ordres dont il ne comprend pas le sens, que parfois même il ressent comme iniques et à l'opposé de son penchant naturel, l'enfant apprend à ne pas suivre sa nature, pourtant souvent plus juste que les règles des adultes; il apprend à se méfier de lui-même, à se mépriser et à accorder une confiance aveugle à l'autorité de ses éducateurs : parents, prêtres ou professeurs, et des institutions : État, Église, Armée. 


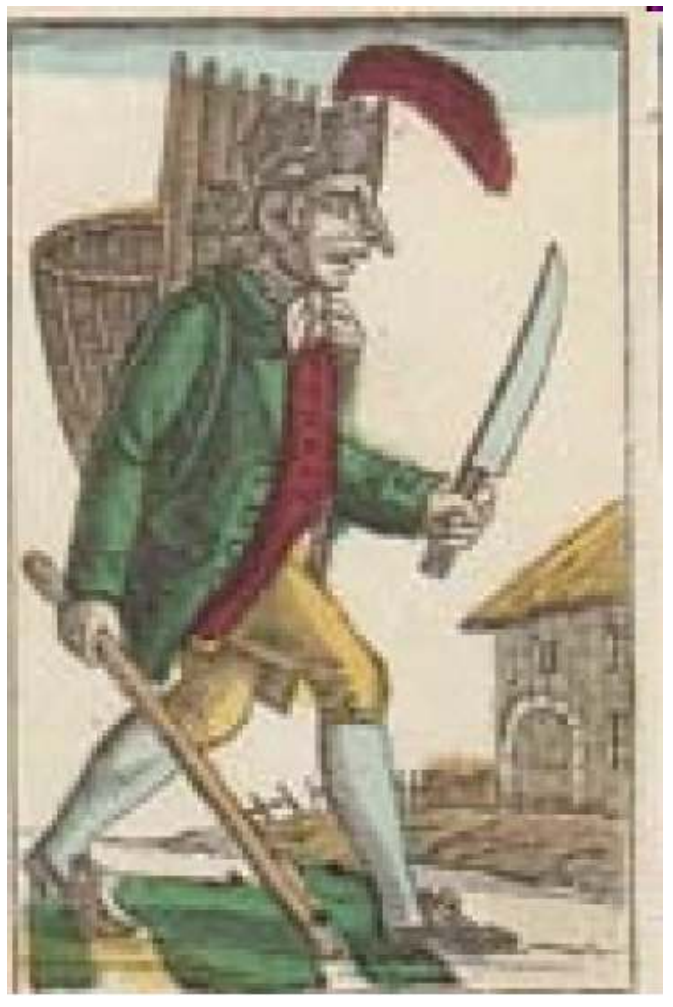

Dans son étude Pédagogie noire 5 (1977), Katharina Rutschky (née à Berlin en 1941) situe elle aussi l'origine du mal dans la famille; dès la plus tendre enfance, avec les contes de fées et les livres de jeunesse, on imprime des images monstrueuses dans la tendre imagination des plus petits : L'enfant incorrigible (Das eigensinnige Kind) des frères Grimm, le Struwwelpeter duD ${ }^{\mathrm{r}} \mathrm{H}$. Hoffmann (1809-1894), et Max und Moritz de Wilhelm Busch (1832-1908). Des personnages, tels que Croque-mitaine, le père Fouettard, ou le loupgarou et autres créatures maléfiques incitent les enfants au respect aveugle de l'ordre établi. Dans une perspective psychanalytique, Ruschky et Miller posent comme point de départ la mise en place d'un sur-moi social et la formation, dans la psyché, d'un mécanisme d'autodéfense qui se durcit au cours de la vie. Comme Foucault, elles dénoncent l'instrumentalisation du corps et de certaines de ses parties en faveur de fonctions sociales prédéfinies. Depuis les Lumières, l'éducation aurait ainsi, selon nos trois auteurs, été réduite à un dressage comme pour les animaux; elle ne favoriserait plus un apprentissage raisonné des mécanismes sociaux et empêcherait l'épanouissement de la personnalité, son but étant la soumission aveugle du sujet à une autorité incontestée. 


\section{Les valeurs transmises par le Struwwelpeter (1844)}

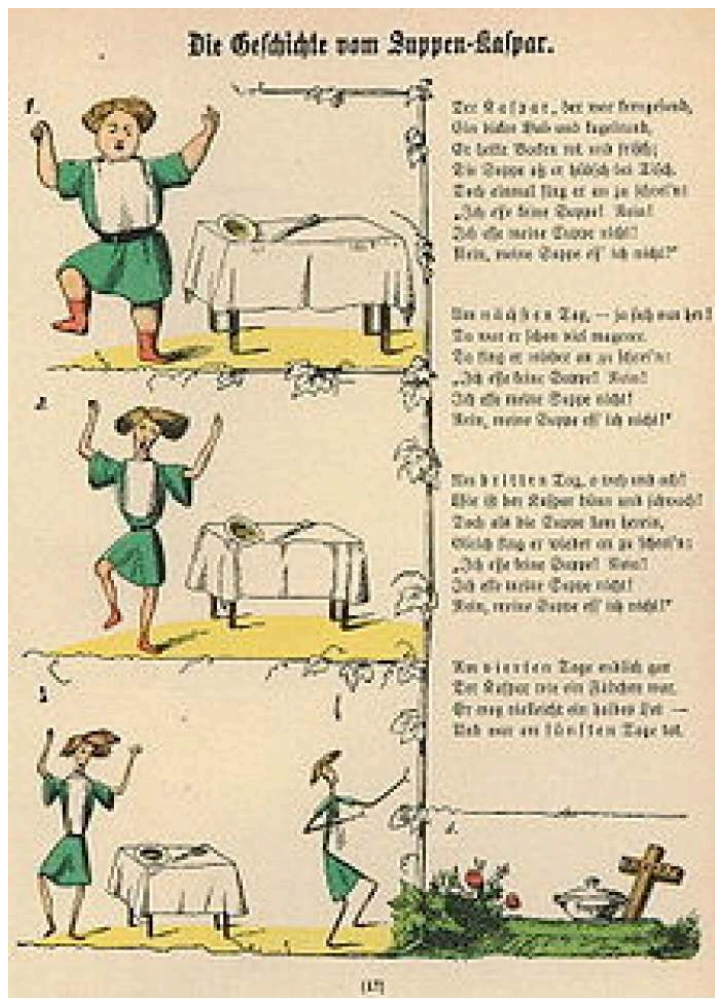

Le Struwwelpeter de Heinrich Hoffmann reste aujourd'hui encore un classique de la littérature enfantine, bien qu'il transmette les valeurs du XIX ${ }^{\mathrm{e}}$ siècle et ses méthodes d'éducation fondées sur l'autorité, la violence et la peur. Dans cet ouvrage en effet, tout tourne autour du comportement social. Certes, aucune violence physique n'est directement exercée; ni fouet ni bâton ne viennent infliger une juste correction au délinquant, comme la comtesse de Ségur aimait à le faire. La méthode est bien pire, car elle ne laisse aucune place à une juste révolte : tout acte ne respectant pas les normes morales de la pudeur, du maintien et de la politesse, ou tout simplement celles de la prudence, de la concentration et de la maîtrise de soi, est réprimé de la façon la plus cruelle car inscrite dans sa nature même. Hoffmann met la bande dessinée au service d'un programme et d'une image préconçue du bonheur possible uniquement grâce à la raison normative. Contrairement à l'idéal rousseauiste, l'homme n'est pas naturellement bon, c'est l'éducation qui le rend tel. On ne peut pas dire non plus qu'il soit mauvais ; il est ignorant et l'éducateur doit lui présenter les dangers qu'il encourt. Sous ses airs neutres, rationnels et en apparence non marqués idéologiquement, Hoffmann reproduit les mœurs de son époque; perçu comme un adulte en devenir, l'enfant doit cesser de sucer son pouce, se couper les ongles et respecter les interdits : ne pas jouer avec le feu, ne pas se moquer d'autrui, respecter les animaux, surtout enfin obéir à ses parents, car même en leur absence, l'autorité veille. L'innocent bambin suçant son pouce dès que sa mère a le dos tourné, se voit ainsi amputé d'un doigt par un tailleur surgi d'on ne sait où. Paulinchen joue avec les allumettes, malgré l'interdiction formelle de ses parents, et se retrouve réduite à un petit tas de cendre sous le regard moralisateur de ses chats. S'agit-il d'une juste punition ou d'un jeu macabre? 


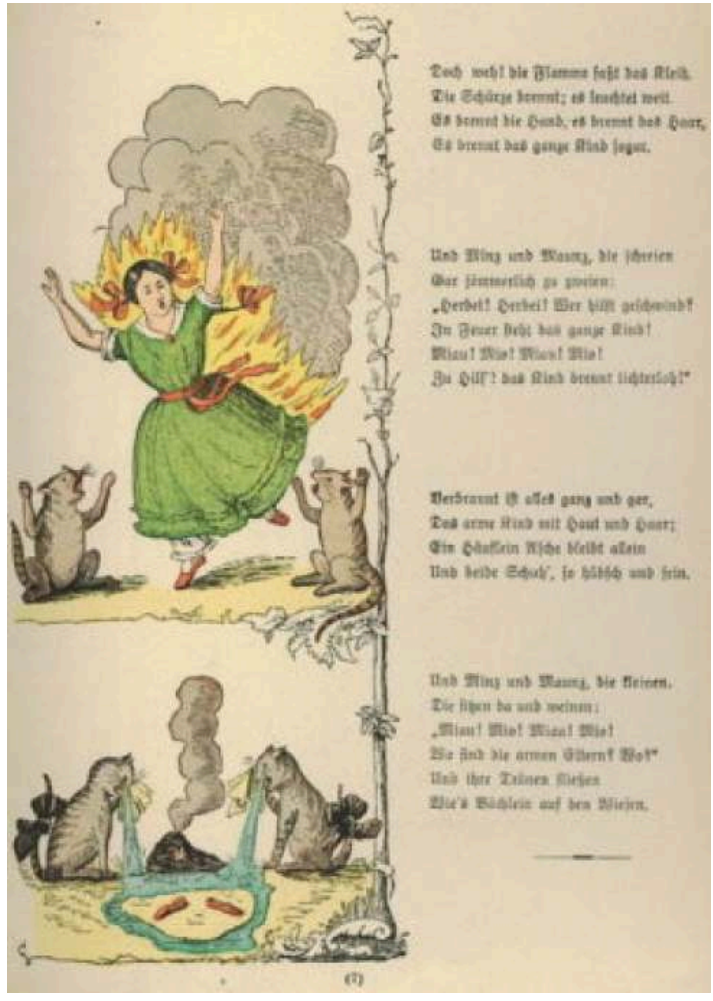

11 Le jeune lecteur peut-il ne point s'apitoyer sur le destin d'une jeune fille à laquelle il devrait logiquement s'identifier? Comment accepte-t-il sans frémir une sanction aussi terrible? L'excès même du châtiment le protègerait-il dans son invraisemblance en suggérant qu'il en est exclu? Rien n'est moins sûr, tant l'intention pédagogique d'Hoffmann saute aux yeux. Hoffmann ne suggère qu'une seule issue : l'obéissance! Ses anecdotes, qui ne sont d'ailleurs pas des contes, mais plutôt des exemples moraux, mettent l'accent sur la sanction et celle-ci n'a rien de ludique. Les instances vengeresses sont tirées du quotidien proche de l'enfant: le tailleur, le père, tous les adultes qu'il doit apprendre à respecter; ceux-ci incarnant la sagesse, il leur doit obéissance. Avec Saint Nicolas et Jésus, la religion vient enfin au secours des instances civiles. 


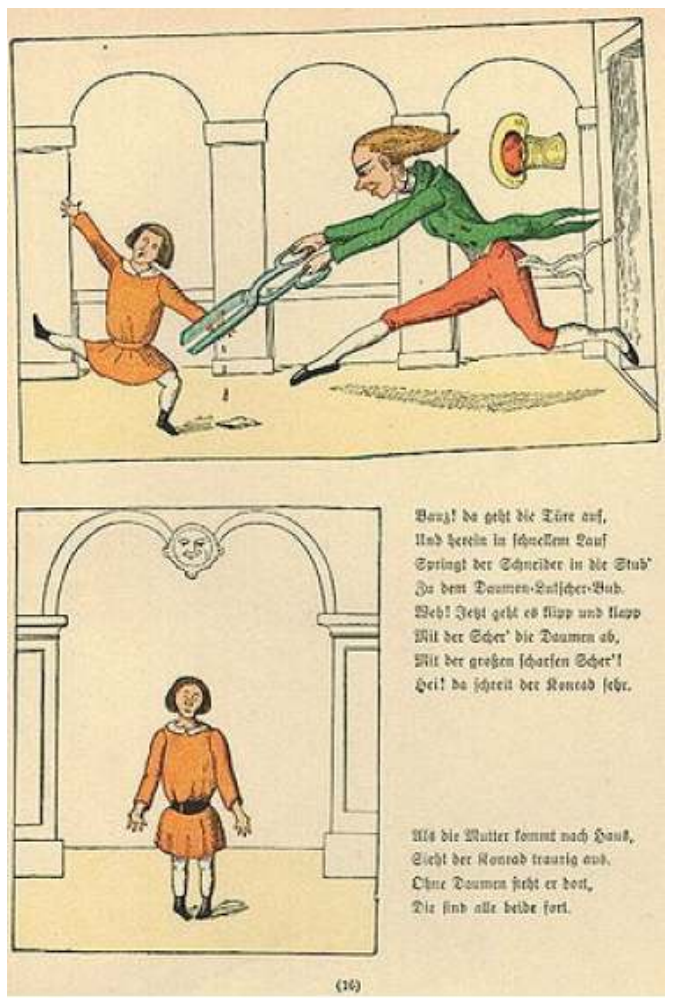

L'inversion des situations dans les fables du Méchant Frédéric et dans L'histoire bien triste des allumettes, en faisant des animaux les représentants de la sagesse, pourraient amuser un ami des chiens et des chats; celui-ci s'identifierait alors aux animaux et non point aux chenapans qui, par leur désobéissance à leur parents, contreviennent à l'ordre établi, accepté comme juste. En réalité, les messages de sagesse, de respect du plus faible et de prudence, transformés en leçons d'une excessive sévérité, suscitent plutôt la terreur. L'histoire du Suppenkaspar qui refuse de manger sa soupe, se termine ainsi de la façon la plus effroyable pour l'imagination encore très réceptive d'un lecteur à peine âgé de 3 à 6 ans : plus que pour ne pas avoir mangé sa soupe, Gaspard meurt en réalité parce qu'il a refusé d'obéir à son père.

Or l'identification, comme Bruno Bettelheim l'a souligné en 1976 dans Psychanalyse des contes de fées ${ }^{6}$, constitue un principe essentiel chargé de réduire la nocivité des contes, tels que ceux des frères Grimm ; mais cet apaisement n'a lieu pour Bettelheim, que dans le cas précis où la cruauté et la violence des événements sont compensés par une fin heureuse. C'est à cette condition seulement qu'a lieu la réconciliation avec l'ordre juste de l'univers; car la fin positive, en rétablissant la justice, rassure l'enfant fasciné et alarmé par les faits souvent horribles que rapportent les contes. Pour Bettelheim, ce n'est pas le triomphe final de la vertu qui assure la moralité du conte mais le fait que, séduit par le héros, le jeune lecteur s'identifie à lui à travers toutes ses épreuves, qu'il partage toutes ses souffrances et qu'il triomphe avec lui au moment où le bien l'emporte sur le mal; c'est seulement grâce à cette identification avec le héros qu'il acquiert le sens moral. Bettelheim ne met donc pas l'accent sur la notion de châtiment mais sur celle d'identification; et c'est pour cela que la fin doit être heureuse. Sinon, l'enfant ne peut pas surmonter son sentiment de frustration et d'insatisfaction ni surtout son angoisse. Or, c'est justement cette fin harmonieuse qui fait défaut chez Hoffmann. 

une jeune imagination ? La stylisation du dessin créerait-elle une distance, s'adressant plus à la raison qu'à l'imagination? Hoffmann promet à son lecteur des « histoires drôles». Or, l'image qui, à première vue, pourrait susciter le rire par la réduction grotesque du dessin à des lignes pures et à des couleurs vives plutôt chaudes, se révèle à l'analyse d'une extrême violence. Aucun élément apaisant ne permet la réconciliation. Certes, la vision des chats en larmes qui semblent inverser la situation habituelle où l'animal subit les sévices des garnements insensibles, peut prêter à sourire... Assurément, les couleurs claires, la simplification caricaturale des visages et des corps, l'aspect grotesque de la soupière posée près de la croix sur la tombe de Gaspar, les larmes qui coulent abondamment des yeux des chats jusqu'à former un ruisseau circulaire, tous ces éléments de la satire, diminuent l'impression de réalité, donc la possibilité d'identification, et réduisent la négativité du message. l'on en croit Bettelheim, le conte, aussi noir fût-il, permettrait à l'enfant d'explorer sans danger les profondeurs de son inconscient. Il ne ferait qu'exprimer en un langage clair et sans ambiguïté les angoisses qui l'habitent. Peut-on partager cette analyse pour le Struwwelpeter? Non, sans aucun doute. Contrairement aux contes de fées souvent merveilleux et situés dans un univers lointain, inaccessible, sans aucun lien avec le quotidien familier à l'enfant, Hoffmann choisit des situations banales et bien connues de son fils dans le but affiché de l'éduquer. Et cette éducation est fondée sur la peur du châtiment.

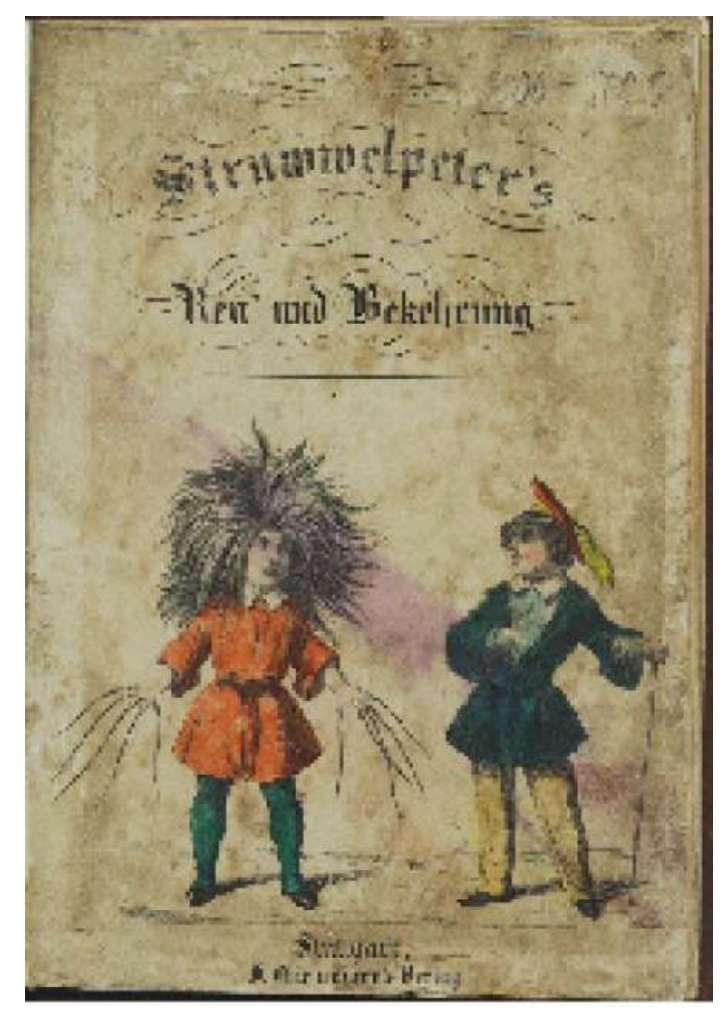

Nombreuses seront les réécritures au cours du xix ${ }^{\mathrm{e}}$ siècle. Dans l'édition de 1851, le dessin plus réaliste acquiert peu à peu une complexité qui lui ôtera toute sa dimension caricaturale pour en faire un véritable tableau' ${ }^{7}$. L'intention moraliste y est encore plus lourdement soulignée. La mère utilise ainsi une scie pour couper les ongles trop longs de l'enfant. Et comme la page de garde l'indique, le but est bien de civiliser le petit tigre 
du début pour en faire un écolier élégant et studieux. L'enfant considéré comme une matière brute, un petit animal sauvage à la chevelure folle, doit entrer le moule: il apprend à se laver, à se couper les cheveux, à se coiffer, à soigner ses vêtements, à tenir la main de sa mère lorsqu'il marche dans la rue, à ne pas courir comme son corps le désire, mais à marcher lentement, à ne point grimper aux arbres, et à rester bien droit et rigide, à manger ce qu'on lui sert, même lorsque son corps n'en ressent pas le besoin; car le corps aussi doit se plier aux normes. Il apprend, comme le dirait Alice Miller, à se soumettre à un code social vidé de son contenu, non point parce qu'il en reconnaît le bien fondé mais parce qu'il a peur d'un châtiment disproportionné.

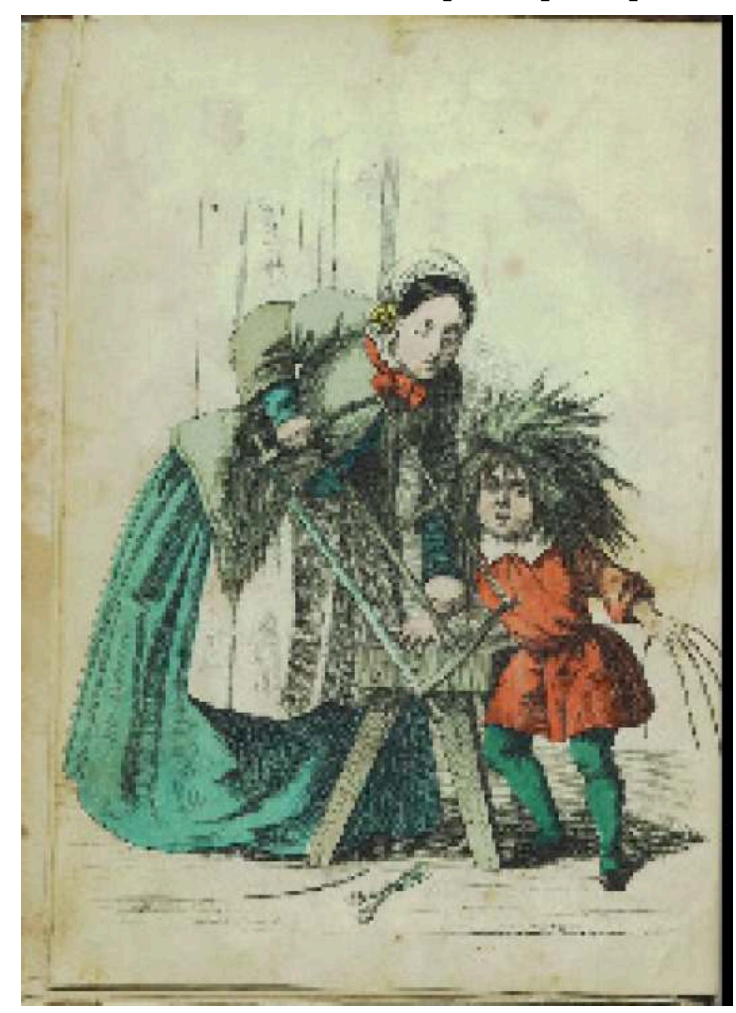

La leçon de prudence, de sagesse, et de bonne conduite du premier Struwwelpeter prend un caractère civilisateur à la fin du siècle. Avec la scolarisation obligatoire et la réforme humboldtienne du système éducatif, tous les enfants du peuple doivent désormais adopter une tenue vestimentaire et une hygiène leur permettant de se fondre dans la masse des écoliers. Ce qui, au XIx ${ }^{\mathrm{e}}$ siècle apparaît comme un progrès permettant à tous d'accéder à la culture par l'éducation, devient au $\mathrm{xx}^{\mathrm{e}}$ une leçon de conformisme ; la génération des années 1960-1970, tourmentée par le souvenir pesant de la massification et l'uniformisation du monde pendant la période fasciste, fustige désormais la vision d'un homme prisonnier du vêtement, des lois et des traditions. 


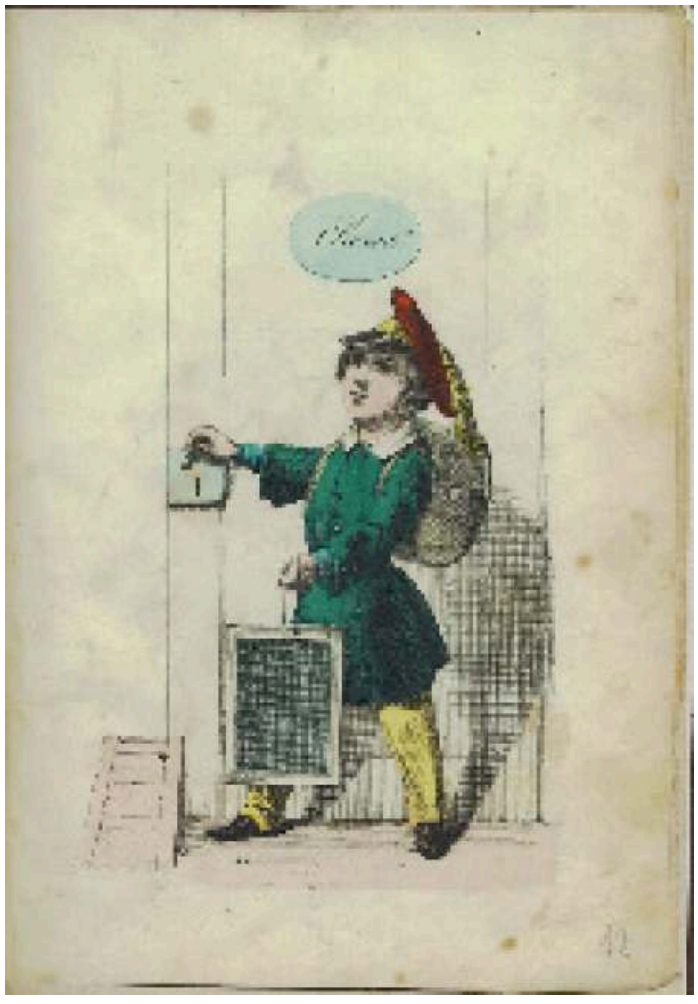

\section{Der Anti-Struwwelpeter ${ }^{8}$ ou l'imagination au pouvoir !}

La jeunesse s'enthousiasme alors pour une forme de vie alternative rompant avec le modèle familial, le couple fermé sur soi et l'éducation autoritaire des générations précédentes. Au corps maîtrisé décrit par Foucault, on oppose le corps libéré de toute contrainte, à l'immobilité sage, le désordre du mouvement libre, la nudité, la danse. La crinière du Struwwelpeter au regard triste, devient un symbole de joie et de sensualité sur la couverture de l'Anti-Struwwelpeter au sourire large et au regard malicieux. Une simple mise en parallèle des pages d'introduction suffit à faire éclater le changement des mentalités : à la peinture bien ordonnée, bien symétrique d'Hoffmann s'oppose la vision désordonnée des adultes grimaçant, perdant l'équilibre et tombant à la renverse sur la planche que tient le petit garnement sur ses frêles épaules. Chez Waechter, le point de vue est celui de l'enfant: les adultes sont grotesques, incompétents ; l'enfant est maître de leur destin; son regard, dirigé du bas vers le haut, provoque la déformation de leur corps. C'est la perspective du gnome, ou plutôt du lutin dont la plaisante candeur fera surgir le bien dissimulé derrière la carapace des mauvaises habitudes. 


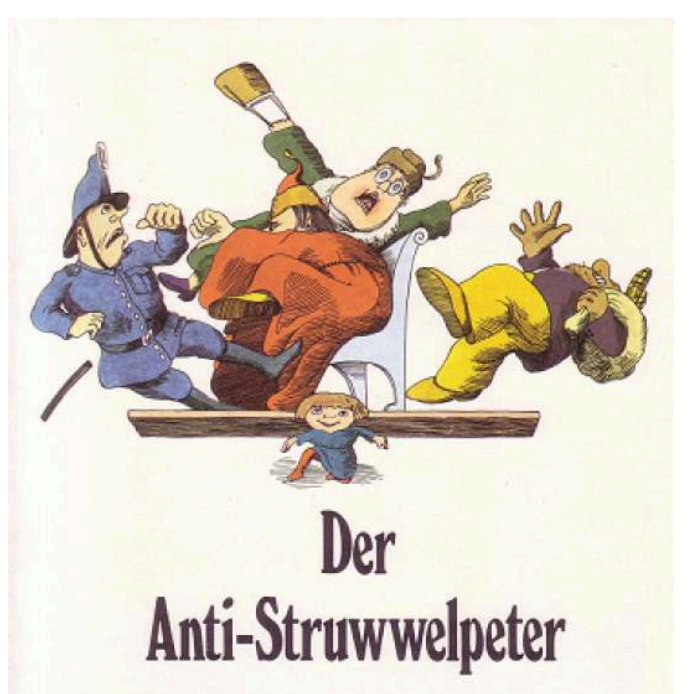

Text und Illustrationen

von Friedrich Karl Whechter

\section{MelzerVerlag}

19 Chez Hoffmann, inversement, la perspective du récit est celle de l'adulte. Le tableau mis en exergue exprime l'ordre, l'équilibre la rationalité, l'univers paisible de ceux qui jouent en silence dans leur chambre, mangent sagement leur soupe, et tiennent gentiment la main de leur mère dans la rue. Seuls ceux-là ont droit à la générosité de Jésus le jour de Noël. Dans le ciel étoilée, - les étoiles sont le regard de Dieu, disait Maeterlinck - l'ange aux ailes multicolores bien centré en haut du tableau harmonieusement construit selon une symétrie rigoureuse, confère une douce sérénité à l'ensemble. Les chérubins déversent les cadeaux en une pluie généreuse sur les enfants obéissants. Les illustrations encadrent le texte qu'elles paraphrasent. À la régularité des vers correspond l'équilibre sévère de la composition graphique. Chaque objet est à sa place, chaque être, comme aseptisé, incarne non point un caractère individuel mais un comportement présenté comme bon. Le tableau reste statique, rigide comme l'idéologie qu'il représente. 


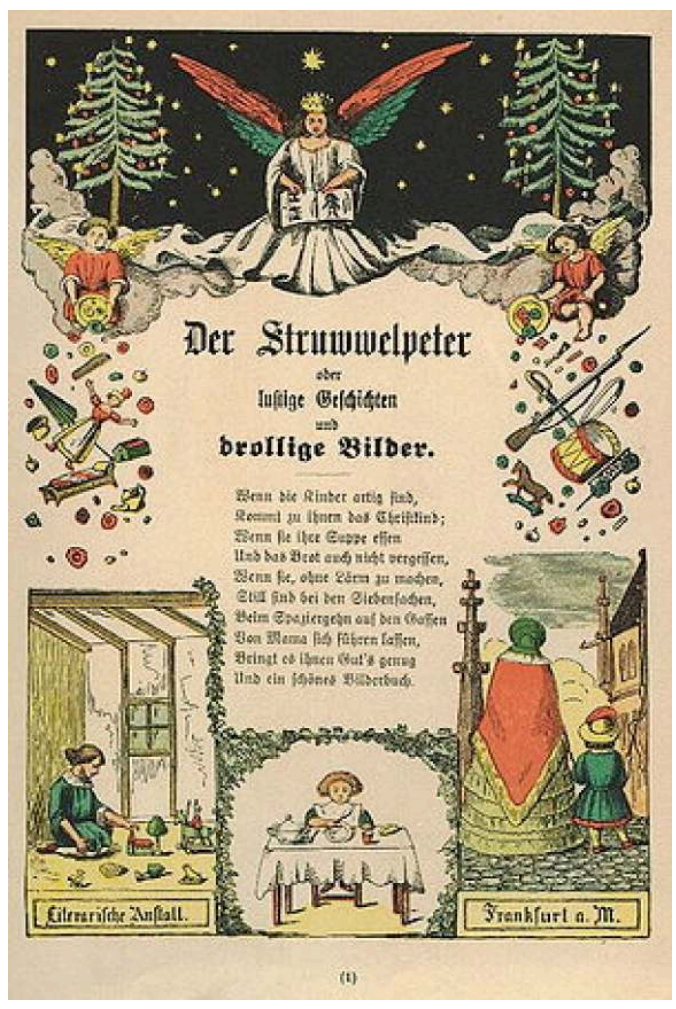

20 À l'opposé, les dessins de Waechter sont tout en mouvement, exprimant le désordre et le déséquilibre. La première planche de l'Anti-Struwwelpeter souligne l'intolérance des adultes prisonniers de préjugés ancestraux sur l'apparence physique et la mode. Privés d'imagination, ils sont vêtus de gris, se trémoussent dans l'ombre au bas de la page, tandis que le petit polisson à l'abondante crinière rousse - on sait que les roux sont, selon une tradition populaire allemande, les créatures du diable -, bien à l'aise dans son costume coloré, domine la composition. Avec ses boucles flamboyantes, sa chemise d'un rouge éclatant et ses pantalons verts, le garçonnet constitue certes une citation exacte de l'original hoffmannien mais il adopte la position dominante; et le visage triste du Struwwelpeter fait place au large sourire de son antimodèle. Ce n'est plus l'ange du seigneur qui déverse ses dons sur l'enfant sage et soumis; c'est le diablotin qui triomphe de l'univers morne et gris des adultes. Les corps bouffis, obèses des adultes forment un contraste saisissant avec le malicieux garnement tout en souplesse, légèreté et force, vainqueur de l'espace et du temps, habitant les cieux, amoureux des nuages.

Pour souligner l'universalité de l'intolérance, Waechter pose une pyramide et un joyeux crocodile sur les bords du Rhin. L'amalgame fait entre la fable de Paulinchen qui n'écoute pas les sages conseils de ses chats, et celle du petit Maure, transforme la sévère leçon de morale en un jeu charmant. Ce qui été conçu comme une terrible punition par Hoffmann: la couleur noire de la peau, devient la source d'un grand bonheur chez Waechter, puisque grâce au plongeon dans l'encrier, Paulinchen peut enfin jouer en toute liberté avec le petit Maure. Victime de ses préjugés racistes, le père n'a pas compris la générosité naturelle de sa fille. Ce n'est plus l'enfant, spontanément généreux et curieux, qui est source de désordre mais le père prisonnier des stéréotypes. Et pour échapper à son autorité, les jeunes héros Max, Frédéric, Maure et Paulinchen partent dans un Kinderladen, où ils pourront s'épanouir dans une ambiance antiautoritaire, ce Kinderladen qu'il faut opposer à l'image finale du Struwwelpeter de 1851, où le petit garçon, les cheveux et les ongles coupés, bien propre après un bain 
d'eau savonneuse, vêtu du bel uniforme et la casquette bien centrée ouvre la porte de l'école, digne enfin de pénétrer dans le temple de la culture.

C'est bien cela que Waechter condamne : l'uniforme, qui, comme celui de son policier bedonnant, symbolise la maîtrise du corps et avec elle le carcan de la tradition; son dessin tout en mouvement, contrairement au style statique de 1851, oppose le corps raide et difforme du gardien de l'ordre à la nudité innocente et naïve des enfants qui l'entourent nus dans une ronde joyeuse. Waechter choisit lui aussi le style de la caricature pour transmettre un idéal qu'il situe hors de la réalité quotidienne. À la rigidité, il oppose la mobilité, à l'uniforme la nudité, à la soumission l'autonomie de la pensée, à la discipline scolaire le jeu sans contrainte.

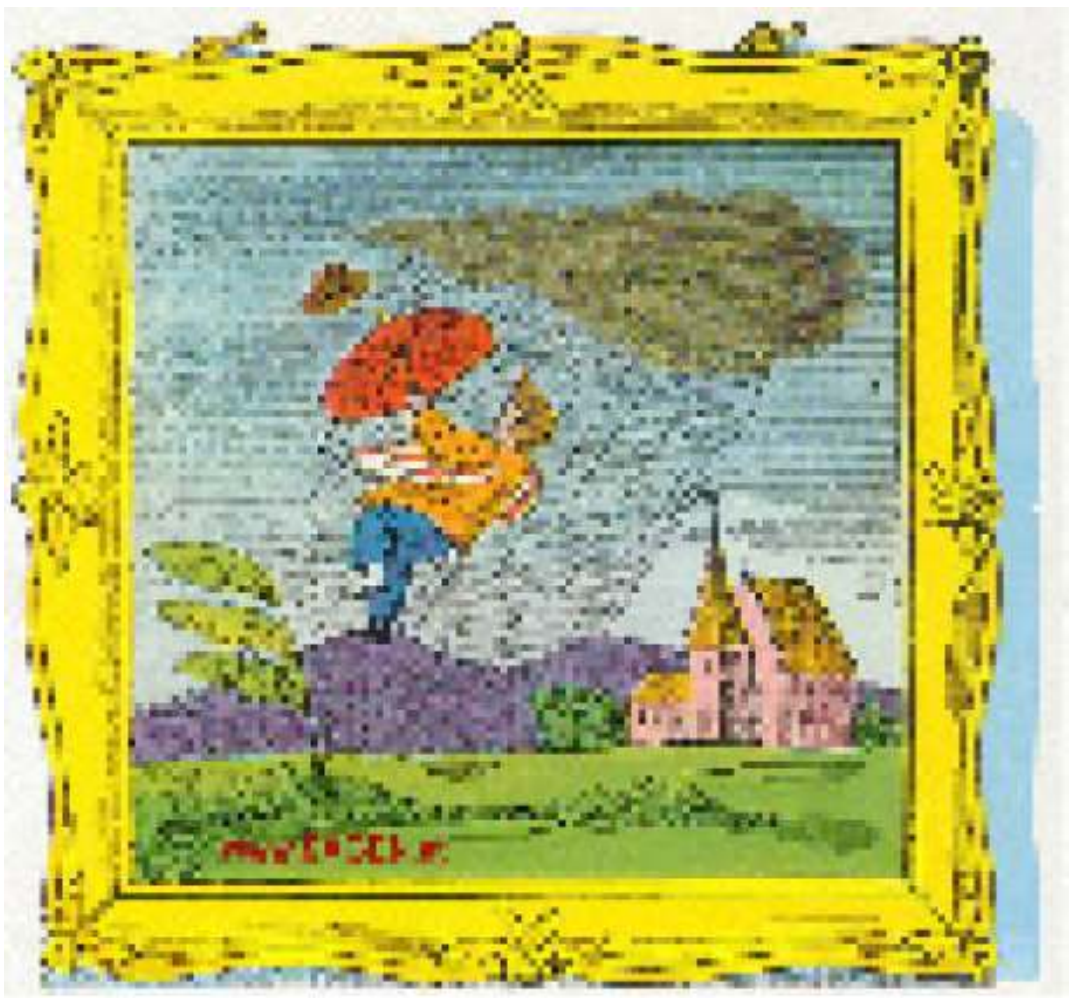

Enfin, en réaction au message moralisateur $\mathrm{du} \mathrm{XIX}^{\mathrm{e}}$ siècle, Waechter établit une séparation nette entre l'univers des grandes personnes qui ne représentent plus ni la raison ni la sagesse, et celui des enfants qui, dans leur spontanéité naturelle, détiennent les clefs du bonheur. Autoritaires, sclérosés, bouffis et bornés, les adultes ne pensent qu'en termes de rentabilité ou de profit, et défendent les valeurs du capitalisme. Les enfants de leur côté, ignorant les contraintes économiques se laissent emporter par leur imagination créatrice ; eux seuls savent transformer le monde avec leurs rêves; et ce sont eux qui désormais éduquent les adultes en leur apprenant à s'accepter euxmêmes, à se "décoincer ", à surmonter leurs peurs et à renoncer au matérialisme qui tue leur fantaisie. Le rêve et l'imagination finissent par vaincre l'esprit mercantile et le " réalisme » des adultes. Si Robert Volant, puni par son imprudence et sa désobéissance disparaît emporté par la tempête, le Robert de Waechter s'enivre en planant dans les airs. Victime, dans un premier temps, de son père qui ne voit en son art qu'un moyen d'enrichissement, il finit par entraîner son géniteur dans les cieux pour célébrer le triomphe du rêve et de la créativité sur la cupidité. Comme dans L'Anti-đEdipe de Gilles Deleuze $^{9}$, ce n'est point le fils qui assassine le père, mais le père, c'est-à-dire le capitalisme. 
Waechter ne procède pas à une simple inversion des valeurs : l'esprit hiérarchique fait place à une heureuse complicité. Le livre se termine en effet sur la réconciliation des enfants et des adultes, chacun pouvant apprendre de l'autre. Ce qui triomphe, c'est l'esprit de découverte, la créativité, les droits d'une nature considérée comme bonne et n'ayant nul besoin d'être maîtrisée.

En conclusion, la mise en parallèle des deux bandes dessinées, sans oublier le livre d'images de 1851, fait apparaître deux conceptions de l'homme radicalement opposées : si Hoffmann et ses successeurs ne conçoivent l'enfant que comme une matière brute, sauvage, qu'il convient de civiliser, Waechter et les adeptes de l'éducation antiautoritaire fondent leur espoir sur la nature fondamentalement bonne de l'humanité. Avec Deleuze, Foucault, Miller et Rutschky, Waechter condamne les normes civilisatrices qui ont perverti cette nature qu'il convient désormais de reconquérir. Chez Waechter, l'adulte doit retrouver la spontanéité perdue. Et l'on pourrait dire que son livre ne s'adresse en réalité pas aux enfants mais bien à leurs parents ! Quittant le champ de l'enfance, la bande dessinée devient chez lui un pamphlet politique.

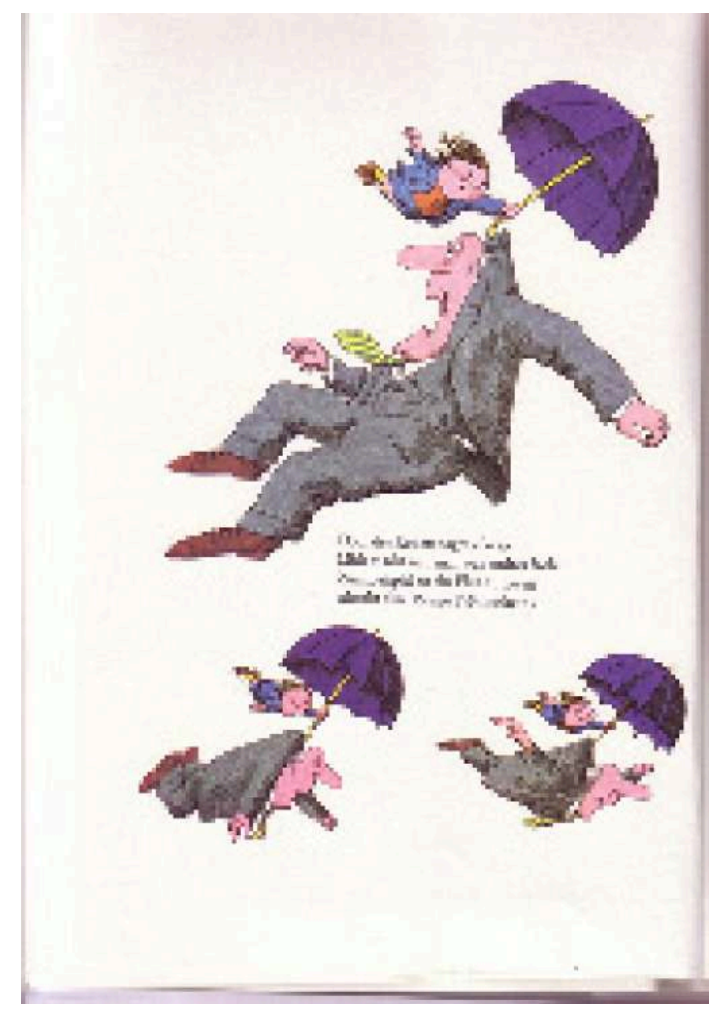

\section{NOTES}

1. Johannes Pommeranz, Hrsg., Struwwelpeters Welt : mit originalgetreuem Nachdruck des « Struwwelpeter»-Urmanuskripts von $D^{\mathrm{r}}$ Heinrich Hoffmann (1809-1894) zu seinem 200. Geburtstag / bearb. von Germanisches Nationalmuseum, G. Ulrich Großmann. Ed. Betreuung: Christine Kupper... Kunsttechnologische Betreuung: Frank Heydecke. Nürnberg: Verl. des Germanischen 
Nationalmuseums 2009. Detlev Gohrbandt: Das Pädagogische und das Politische. Wandlungen der Suppenkaspar-Geschichte in englischen Struwwelpeter-Satiren. In: Dietrich Grünewald (Hg.): Struktur und Geschichte der Comics. Beiträge zur Comicforschung. Christian A. Bachmann Verlag, Bochum 2010.

2. Friedrich Karl Waechter, Der Anti-Struwwelpeter,Frankfurt am Main, Joseph Melzer Verlag, 1970.

3. Michel Foucault, Surveiller et punir, Paris Tel Gallimard, 1998, pp. 234-235.

4. Alice Miller, Am Anfang war Erziehung, 1980. trad. fr. par J. Etoré, C'est pour ton bien, Paris Aubier 1983.

5. Schwarze Pädagogik. Quellen zur Naturgeschichte der bürgerlichen Erziehung. Herausgegeben und eingeleitet von Katharina Rutschky. 8. Aufl. München 2001. [Erstausgabe: Frankfurt a.M. u.a. 1977.].

6. Bruno Bettelheim, (1903-1990), Psychanalyse des contes de fées, traduit de l'américain par Théo Carlier, Paris, Club français du livre, 1976. The Uses of Enchantment: The Meaning and Importance of Fairy Tales, Knopf, New York, 1976.

7. Struwwelpeter's Reu und Bekehrung. Allen Kindern zur Lust u. Belehrung in Bild und Reim gebracht. Stuttgart Thienemann, 1851. Le livre et a été numérisé par bibliothèque de Braunschweig et se trouve donc sur l'Internet. http://rzbl04.biblio.etc.tubs.de:8080/docportal/servlets/ MCRFileNodeServlet/DocPortal_derivate_00004311/1006-9785.pdf.

8. Friedrich Karl Waechter, Der Anti-Struwwelpeter oder listige Geschichten und knallige Bilder, Frankfurt, Melzer, 1970.

9. Gilles Deleuze, Felix Gattari, Capitalisme et schizophrénie, l'Anti-〔Edipe, Paris Les éditions de minuit 1972-73.

\section{RÉSUMÉS}

Dès la fin de la Seconde Guerre mondiale, une crise de la conscience déchire l'Allemagne, entraînant une transformation profonde des mentalités. Après une première phase où la réflexion morale condamne en bloc la nation allemande grâce au concept chrétien de « culpabilité collective» (Kollektivschuld), les intellectuels s'interrogent sur les causes profondes d'une telle débâcle de toutes les valeurs humanistes au cours du national-socialisme. Ecrivains, sociologues, psychologues, pédagogues et philosophes donnent chacun un éclairage divers permettant de comprendre et de traiter le mal à la racine. À la fin des années 1960, la révolte des étudiants aboutit au mouvement de libération connu sous l'étiquette simplificatrice de 1968 ; la jeunesse fustige les structures autoritaires de la société allemande et de sa plus petite cellule : la famille. Tous adhèrent au cri de rage lancé par André Gide dans les Nourritures terrestres: «Familles, je vous hais! » La réécriture du Struwwelpeter de Heinrich Hoffmann (1809-1894) par Friedrich K. Waechter (1937-2005) se situe dans ce contexte. Le livre qui a connu un succès incontesté jusqu'à aujourd'hui, devient la cible des attaques de la génération libertaire des années 1970. Friedrich K. Waechter (1937-2005), dessinateur et caricaturiste des revues gauchistes Pardon et Twen enréécrit une nouvelle version anti-autoritaire : Der Anti-Struwwelpeter. Proche de la Kommune I (1967-1969), il partage son mépris des structures familiales et sociales accusées de brider l'imagination et la créativité naturelles à l'homme. Une comparaison des deux œuvres fait apparaître le renversement des valeurs qui a eu lieu dans la deuxième moitié du xx 
siècle en Allemagne, le principe d'autorité étant remplacé par les droits de l'imagination créatrice.

Nach dem Zweiten Weltkrieg bekannten sich viele Intellektuelle zur kollektiven Verantwortung des deutschen Volkes. Zuerst wurde diese Frage nach einer kollektiven Schuld als eine moralische Forderung verstanden, aber bald verließ man die theologisch-moralische Ebene, um nach den Ursachen des Nationalsozialismus zu suchen. Die Studentenbewegung Ende der 1960er Jahre meinte in den autoritären Strukturen von Familie und Gesellschaft den Urgrund eines solchen Debakels aller humanistischen Werte zu finden. Dazu gehörte die Kritik an den auf Angst und Zwang gründenden Erziehungsmethoden der Vergangenheit und an der Jugendliteratur, die die Werte des Gehorsams und der blinden Autoritätshörigkeit vermittelte. Hierzu ist die Neufassung von Heinrich Hoffmanns (1809-1894) Struwwelpeter durch Friedrich K. Waechter (1937-2005) zu rechnen. Friedrich K. Waechter (1937-2005), der Karikaturist der linken Zeitschriften Pardon und Twen,lieferte eine neue Interpretation des Struwwelpeter und verfasste eine antiautoritäre Version des Jugendklassikers: DerAnti-Struwwelpeter. Mit der Berliner Kommune I (1967-1969) kritisierte er die familiären und gesellschaftlichen Strukturen, weil sie die Kreativität und die gesunde Entwicklung eines freien und selbstbewussten Bürgers verhindern würden.Ein Vergleich zwischen beiden Werken deckt den Mentalitätswandel und die grundverschiedene Haltung zu Kindheit und Jugend zwischen dem 19. und dem Ende des 20. Jahrhunderts auf.

After the Second World War, Germany was gripped by a crisis of conscience which produced a profound and widespread shift in thinking. After a first period of moral reflection which led to outright condemnation of the whole German nation, under the Christian notion of « collective blame » (Kollektivschuld), intellectuals set out to question the underlying causes of the collapse of all human values under National Socialism. Writers, sociologists, psychologists, academics and philosophers all cast different light on ways of understanding and uprooting the problem. In the late 1960s, student unrest gave birth to the libertarian movement reductively labelled " 1968 ». Young people attacked the authoritarian structures of German society and of its core unit, the family. They subscribed to André Gide's angry outburst in Les Nourritures terrestres, «Familles, je vous hais!»: «Families, I hate you!». The rewriting of Heinrich Hoffmann's (1809-1894) Struwwelpeter by Friedrich K. Waechter (1937-2005) should be seen in this context. The original book, whose uninterrupted success continues to this day, became a target for attack of the 1970s libertarian generation. Waechter was an illustrator and cartoonist for the leftist periodicals Pardon and Twen and in the late 1960s had been close to the group Kommune I. His AntiStruwwelpeter reflected contempt for the traditional family and social structures which were held responsible for constricting innate human creativity and imagination.

\section{AUTEUR}

\section{MARIE-HÉLÈNE QUÉVAL}

Université du Maine (Le Mans) 